\title{
Dinámica poblacional, estructura urbana y producción del espacio habitacional en la zona metropolitana de la ciudad de México
}

\section{Martha Schteingart*}

En este trabajo se intenta explicar algunas tendencias del crecimiento y la distribución de la población, y de la estructuración del espacio en la zona metropolitana de la ciudad de México, relacionando datos censales, que han servido como indicadores para medir los procesos de crecimiento y consolidación urbana (a nivel de las unidades político-administrativas que forman parte de la $\mathrm{ZMCM}$ y de los contornos que se constituyen con la adición de esas unidades) con la información obtenida de organismos oficiales, $y$ los propios datos de la autora referidos a la producción del espacio habitacional. Entre las conclusiones importantes del trabajo se destacan las que tienen que ver con el gran crecimiento de algunas unidades politico-administrativas y su relación con las prácticas de los promotores inmobiliarios privados (articuladas, muchas veces, a las políticas y programas de organismos habitacionales del Estado) así como las que relacionan el crecimiento y la expansión urbanos con el desarrollo de espacios habitacionales, tanto dentro del llamado sector "formal" como del sector "informal" de la vivienda.

\section{Introducción}

Hemos analizado la estructura espacial metropolitana en algunos trabajos más o menos recientes (Rubalcava y Schteingart, 1985, 1987); aquí haremos un pequeño resumen de los mismos y propondremos una nueva forma de observar la organización espacial, agregando algunas consideraciones de tipo explicativo que se relacionan con la producción del espacio construido habitacional .

En los trabajos mencionados hemos estudiado concretamente los grandes lineamientos de la estructura de la zona metropolitana de la ciudad de México (ZMCM), describiendo de manera sistemática los fenómenos de la diferenciación socioespacial y su evolución de 1950 a 1980. En ese análisis utilizamos información censal y seleccionamos las variables fisicoespaciales y socioeconómicas que permitieran, a partir de la construcción de un índice que las reflejara (usando el análisis factorial), medir esas diferencias en la ZMCM.

* Profesora-investigadora del Centro de Estudios Demográficos y de Desarrollo Urbano de El Colegio de México. 
La técnica utilizada permitió que para 1970 y 1980 las ocho variables introducidas (los porcentajes correspondientes de la población económicamente activa, de los trabajadores por cuenta propia, de la población de 12 años y más con educación primaria completa o posprimaria, de la población con ingreso mayor de seis salarios mínimos, de las viviendas propias y con agua entubada dentro de la misma, así como el índice de personas por cuarto y el porcentaje de población urbana) formaran dos factores importantes que expresaban respectivamente la consolidación y la condición socioeconómica del crecimiento urbano. Esos dos factores se presentaron sólo para 1970 y 1980 , ya que por falta de información censal, para 1950 y 1960 apareció únicamente el factor que definimos como expresión de la consolidación urbana, fenómeno que implica el mejoramiento de las condiciones socioespaciales de la ciudad a medida que avanza la urbanización en la periferia. Aquél indicó que las unidades más antiguas de la ZMCM, que hacía más tiempo se habían urbanizado, alcanzaron mejores niveles de servicio y equipamiento - sobre todo en lo referente a la provisión de agua y escuelas - así como mejores condiciones con relación a la ocupación de la vivienda. Luego, para observar con mayor claridad las diferencias intraurbanas, las unidades políticoadministrativas incluidas en cada corte temporal se agruparon en un número menor de zonas, y con ese fin se construyeron índices para calificar a las unidades de acuerdo con su jerarquía en cada factor e integrar zonas lo más homogéneas posible, lo que condujo a la determinación de seis zonas para cada uno de los factores. Éstas nos permitieron mostrar una distribución espacial de los fenómenos estudiados y al mismo tiempo obtener una calificación para cada unidad incluida en la ZMCM.

En los trabajos citados dejámos aclarado que los análisis realizados sólo permitían observar las diferencias que se daban en distintos momentos, pero no inferir las causas de los cambios observados, ni conocer qué elementos habían intervenido para que la situación descrita en un corte temporal haya variado a la correspondiente a otro corte.

Con el objeto, justamente, de dar un comienzo de explicación, trataremos de vincular algunas conclusiones de la mencionada investigación con los avances que hemos realizado en el conocimiento de la producción del marco construido, habitacional sobre todo dentro del llamado, sector "formal" de la vivienda, que comprende particularmente las promociones de los organismos públicos y de las empresas privadas, así como aquellas que resultan de la articulación de las prácticas de ambos. Por supuesto que una parte importante del crecimiento y la expansión urbanos corres- 
ponde a asentamientos irregulares, a los que también nos referiremos, pero de manera marginal, pues no han constituido el centro de nuestras investigaciones. Si bien estamos conscientes de las limitaciones de este análisis, también creemos que hay que comenzar a realizar descripciones globales y explicaciones de conjunto que superen los estudios puntuales o sectoriales, muy limitados, referidos a alguna colonia o un pequeño sector del espacio metropolitano.

Para poder vincular, en un primer momento, las descripciones relativas a la conformación del espacio metropolitano con algunos aspectos de la producción social del mismo, introduciremos el análisis poblacional por contorno, dentro del cual nos ha interesado particularmente clarificar la dinámica del crecimiento de la población de 1950 a 1980 (cuadros 1 y 2). Los contornos se han definido [Negrete y Salazar, 1986] a partir de una serie de variables y del criterio de contigüidad, lo cual ha permitido determinar varios conglomerados, cuya distancia al centro es diferente y que conforman, además del centro, tres coronas o anillos [véanse mapas $1,2,3$ y 4]. Teniendo en cuenta futuras comparaciones de esta metrópoli con otras, preferimos usar el criterio más difundido de anillos y para ello se establece una relación entre éstos y las zonas que se conformaron a partir del trabajo al que nos referimos (ver cuadro 3). Una vez detectadas las unidades políticoadministrativas y los contornos que más han aportado al crecimiento total de la población metropolitana en las décadas que van de 1950 a 1980, trataremos de explorar y evaluar la contribución del sector "formal" de la producción habitacional a la forma que adopta el crecimiento urbano, vinculando asimismo esa información con la definición soçioespacial que habíamos obtenido de la investigación mencionada, y que se aplicaba a cada una de las delegaciones del Distrito Federal así como a los municipios conurbados del Estado de México. La hipótesis que orientó esta investigación se relaciona con el hecho de que una parte mayoritaria del espacio urbano está constituida por zonas habitacionales y que dentro de éstas los fraccionamientos para vivienda han tenido una importancia destacada, sobre todo en el crecimiento periférico. ${ }^{1}$

\footnotetext{
${ }^{1}$ Quisiéramos aclarar que el estudio de los fraccionamientos se limitó a los municipios conurbados del Estado de México porque no se obtuvo una información similar para el Distrito Federa]. Además, es en ellos donde se ha dado el mayor crecimiento de la población en las últimas décadas, y también la mayor expansión de la mancha urbana. Se ha calculado que el desarrollo por medio de fraccionamientos ha representado, de 1958 a 1981 , cerca del $40 \%$ de la superficie de la mancha urbana dedicada a uso habitacional en esos municipios.
} 


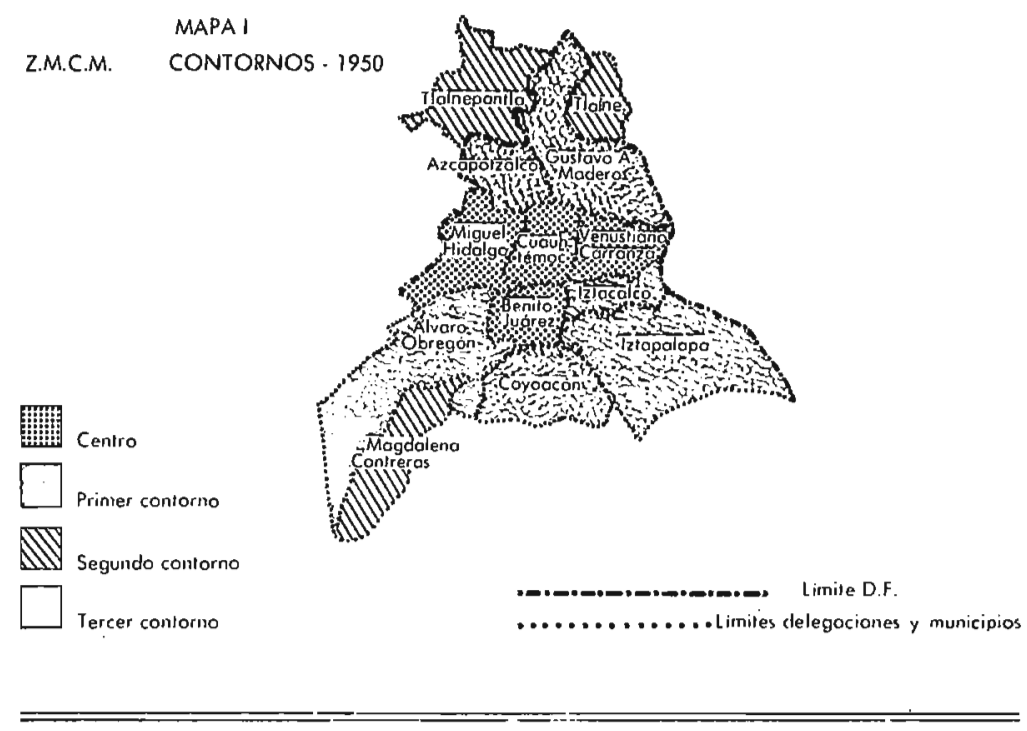

\section{MAPA II}

Z.M.C.M. CONTORNOS $\cdot 1960$
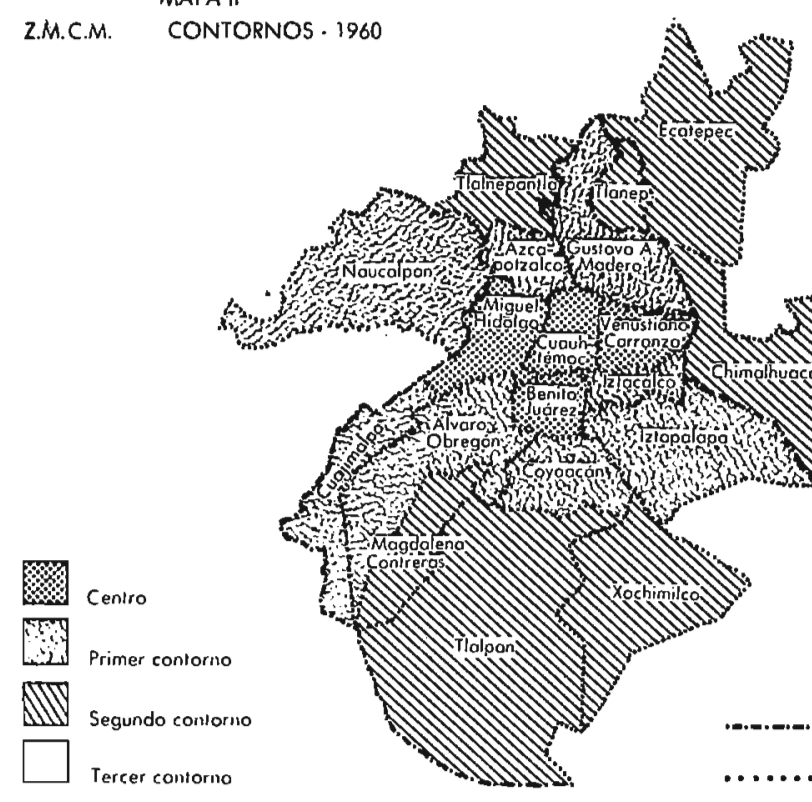
CUADRO 1

Distribución espacial de la población por contorno en la zMCM (1950-1980)

\begin{tabular}{|c|c|c|c|c|c|c|c|c|}
\hline \multirow{2}{*}{$\begin{array}{l}\text { Unidad } \\
\text { política }\end{array}$} & \multicolumn{8}{|c|}{ Población } \\
\hline & 1950 & $\%$ & 1960 & $\%$ & 1970 & $\%$ & 1980 & $0 \%$ \\
\hline \multicolumn{9}{|l|}{ Benito } \\
\hline Juárez & 354298 & & 442374 & & 501363 & & 544882 & \\
\hline Cuauhtémoc & 1042226 & & 980111 & & 853550 & & 814983 & \\
\hline Miguel & & & & & & & & \\
\hline Hidalgo & 442736 & & 661109 & & 656647 & & 543062 & \\
\hline \multicolumn{9}{|l|}{ Venustiano } \\
\hline Carranza & 395535 & & 748539 & & 891409 & & 692896 & \\
\hline Centro & 2234795 & 75.7 & 2832133 & 55.3 & 2902969 & 32.7 & 2595823 & 18.7 \\
\hline Azcapotzálco & 187864 & & 370724 & & 543315 & & 601524 & \\
\hline Coyoacán & 70005 & & 169811 & & 349823 & & 597129 & \\
\hline Cuajimalpa & & & 19199 & & 37212 & & 91200 & \\
\hline \multicolumn{9}{|l|}{ Gustavo A. } \\
\hline Madero & 204833 & & 579180 & & 1223647 & & 1513360 & \\
\hline Iztacalco & 33945 & & 198904 & & 495847 & & 570377 & \\
\hline \multicolumn{9}{|l|}{ Álvaro } \\
\hline Obregón & 93176 & & 220011 & & 471442 & & 639213 & \\
\hline $\begin{array}{l}\text { Nezahual- } \\
\text { cóyotl }\end{array}$ & & & & & 651000 & & 1341230 & \\
\hline Naucalpan & & & 85828 & & 407825 & & 730170 & \\
\hline \multicolumn{9}{|l|}{ Primer } \\
\hline \multicolumn{9}{|l|}{ Magdalena } \\
\hline Contreras & 21955 & & 40724 & & 77478 & & 173105 & \\
\hline Tláhuac & & & & & 64451 & & 146923 & \\
\hline Tlalpan & & & 61195 & & 135105 & & 363974 & \\
\hline Xochimilco & & & 70381 & & 119073 & & 217481 & \\
\hline Atenco & & & & & & & 16418 & \\
\hline Coacalco & & & & & 13197 & & 97353 & \\
\hline \multicolumn{9}{|l|}{ Chimalhua- } \\
\hline cán & & & 76740 & & 18811 & & 61816 & \\
\hline Ecatepec & & & 40805 & & 232686 & & 784507 & \\
\hline La Paz & & & & & 34297 & & 99436 & \\
\hline Tultitlán & & & & & 55161 & & 136829 & \\
\hline \multicolumn{9}{|l|}{ Atizapán de } \\
\hline Zaragoza & & & & & 47729 & & 202248 & \\
\hline Tlanepantla & 29005 & & 105447 & & 387377 & & $778 \quad 173$ & \\
\hline Huixquilucan & & & & & 34604 & & 78149 & \\
\hline \multicolumn{9}{|l|}{ Segundo } \\
\hline
\end{tabular}


CUADRO 1 (continuación)

\begin{tabular}{|c|c|c|c|c|c|c|c|c|}
\hline \multirow[b]{2}{*}{$\begin{array}{l}\text { Unidad } \\
\text { política }\end{array}$} & \multicolumn{8}{|c|}{ Población } \\
\hline & 1950 & $\%$ & 1960 & $0 \%$ & 1970 & $\%$ & 1980 & $\%$ \\
\hline Milpa Alta & & & & & & & 53616 & \\
\hline Cha]co & & & & & & & 78393 & \\
\hline Chicoloapan & & & & & & & 27354 & \\
\hline Chiconcuac & & & & & & & 11371 & \\
\hline Ixtepa]uca & & & & & & & 77862 & \\
\hline \multicolumn{9}{|l|}{ Nico]ás } \\
\hline Romero & & & & & & & 112645 & \\
\hline Tecamac & & & & & & & 84129 & \\
\hline Texcoco & & & & & & & 105851 & \\
\hline Chiautla & & & & & & & 10618 & \\
\hline \multicolumn{9}{|l|}{ Cuautitlán- } \\
\hline Izcalli & & & & & & & 173754 & \\
\hline \multicolumn{9}{|l|}{ Cuatitlán- } \\
\hline R. Rubio & & & & & 41156 & & 39527 & \\
\hline \multicolumn{9}{|l|}{ Tercer } \\
\hline contorno & & & & & 41156 & 0.5 & 775120 & 5.5 \\
\hline Total ZMCM & 2952199 & 100.0 & $5 \quad 125437$ & 100.0 & 8882882 & 100.0 & 13873912 & 100.0 \\
\hline
\end{tabular}

Nota: los datos de las delegaciones centrales se tomaron del Estudio Demográfico, CEED,

El Colegio de México, 1975, para 1950; de Negrete y Salazar (1986) para 1960 y 1970.

Fuente: Censos de Población y Vivienda, 1950, 1960, 1970, 1980.

\section{Resultados del análisis de la información}

Crecimiento y distribución de la población

De 1950 a 1980 la ZMCM ha multiplicado más de cuatro veces su población y ha ido ocupando nuevos territorios tanto en el Distrito Federal como en el Estado de México, donde el crecimiento ha sido más violento, sobre todo a partir de 1960. Además de las cuatro delegaciones centrales [que hasta el censo de 1970 constituían 12 cuarteles), esa zona ha comprendido ocho unidades en 1950, 14 en 1960, 22 en 1970 y 33 en 1980, de las cuales sólo una pertenecía al Estado de México en la primera fecha, cuatro en la segunda, 11 en la tercera y 21 en la última fecha.

La población ha sufrido un fuerte proceso de suburbanización que se aprecia claramente al analizar su distribución por contorno. Así, en 1950 el $75.7 \%$ de la misma se ubicaba en el centro, y el $1.7 \%$ en el segundo con̈torno, mientras que en 1980 sólo el $18.7 \%$ se seguía alojando en las delegaciones centrales, el $22.8 \%$ en el segundo contorno y el $5.5 \%$ en el tercero. Fue a partir der 1970 que el primer contorno comenzó a absorber más del $50 \%$ de la población total de la zona (ver cuadro 1). El centro ha perdido 
CUADRO 2

Crecimiento de la población por decenios en la ZMCM (1950-1980)

\begin{tabular}{|c|c|c|c|c|c|c|}
\hline \multirow{2}{*}{$\begin{array}{l}\text { Unidad } \\
\text { politica }\end{array}$} & \multicolumn{2}{|c|}{$1950-1960$} & \multicolumn{2}{|c|}{ 1960-1970 } & \multicolumn{2}{|c|}{$1970-1980$} \\
\hline & Absoluto & $\%$ & Absoluto & $\%$ & Absoluto & $\%$ \\
\hline Benito Juárez & 88076 & 4.1 & 58989 & 1.6 & 43519 & 0.9 \\
\hline Cuauhtémoc & -62115 & -2.9 & -126561 & -3.4 & -38567 & -0.8 \\
\hline Miguel Hidalgo & 218373 & 10.0 & -4462 & -0.1 & -113585 & -2.3 \\
\hline \multicolumn{7}{|l|}{ Venustiano } \\
\hline Carranza & 353004 & 16.2 & 141870 & 3.8 & -198513 & -4.0 \\
\hline Centro & 597338 & 27.5 & 69836 & 1.9 & -307146 & -6.2 \\
\hline Atzcapotzalco & 182860 & 8.4 & 172591 & 4.6 & 58209 & 1.2 \\
\hline Coyc & 99806 & 4.6 & 180012 & 4.8 & 247308 & 5.0 \\
\hline Cuajimalpa & 19199 & 0.9 & 18013 & 0.5 & 53988 & 1.1 \\
\hline \multicolumn{7}{|l|}{ Gustavo A. } \\
\hline Madero & 374347 & 17.2 & 644467 & 17.2 & 289713 & 5.8 \\
\hline Iztac & 164959 & 7.6 & 296943 & 7.9 & 74530 & 1.5 \\
\hline apa & 177734 & 8.2 & 284322 & 7.6 & 723677 & 14.5 \\
\hline Álvaro Obregón & 126835 & 5.8 & 251431 & 6.7 & 167771 & 3.4 \\
\hline Nezahualcóyotl & & & $651000^{*}$ & 17.3 & 690230 & 13.8 \\
\hline Naucalpan & $85828^{*}$ & 3.9 & 321997 & 8.6 & 322345 & 6.5 \\
\hline \multicolumn{7}{|l|}{ Primer } \\
\hline contorno & 1231568 & 56.7 & 2820776 & 75.1 & 2627769 & 52.6 \\
\hline \multicolumn{7}{|l|}{ Magdalena } \\
\hline Contreras & 18769 & 0.9 & 36754 & 1.0 & 95627 & \\
\hline Tláh & & & $64451^{*}$ & 1.7 & 82472 & 1.7 \\
\hline Tlalpa & $61195^{*}$ & 2.8 & 73910 & 2.0 & 228869 & 4.6 \\
\hline milco & $70381^{*}$ & 3.2 & 48692 & 1.3 & 98408 & 2.0 \\
\hline Aten & & & & & 16418 & 0.3 \\
\hline Coacalco & & & 13197 & 0.4 & 84156 & 1.7 \\
\hline Chimalhuacán & $76740^{*}$ & 3.5 & $-57929^{* *}$ & -1.5 & 43005 & 0.9 \\
\hline Ecate & 40805 & 1.9 & 191811 & 5.1 & 551821 & 11.1 \\
\hline La Paz & & & $34297^{*}$ & 0.9 & 65139 & 1.3 \\
\hline Tultitlán & & & $55161^{*}$ & 1.5 & 81668 & 1.6 \\
\hline \multicolumn{7}{|l|}{ Atizapán de } \\
\hline & & & $47729^{*}$ & 1.3 & 154519 & 3.1 \\
\hline Tlalnepantla & 76442 & 3.5 & 281930 & 7.5 & 390796 & 7.8 \\
\hline Huixquilucan & & & $34604^{*}$ & 0.9 & 43545 & 0.9 \\
\hline \multicolumn{7}{|l|}{ Segundo } \\
\hline & 344332 & 15.8 & 824607 & 22.0 & 1936443 & 38.8 \\
\hline
\end{tabular}

población en el último decenio considerado (1970-1980), sobre todo por el despoblamiento de las delegaciones Venustiano Carranza y Miguel Hidalgo, mientras que en la delegación Cuauhtémoc dicho fenómeno había comenzado desde 1950 (ver cuadro 2). 
CUADRO 2 (continuación)

\begin{tabular}{|c|c|c|c|c|c|c|}
\hline \multirow{2}{*}{$\begin{array}{l}\text { Unidad } \\
\text { política }\end{array}$} & \multicolumn{2}{|c|}{$1950-1960$} & \multicolumn{2}{|c|}{$1960-1970$} & \multicolumn{2}{|c|}{$1970-1980$} \\
\hline & Absoluto & $\%$ & Absoluto & $\%$ & Absoluto & $\%$ \\
\hline Milpa Alta & & & & & $53616^{*}$ & 1.1 \\
\hline Chalco & & & & & $78393^{*}$ & 1.6 \\
\hline Chicoloapan & & & & & $27354^{*}$ & 0.5 \\
\hline Chiconcuac & & & & & $11371^{*}$ & 0.2 \\
\hline Iztapaluca & & & & & $77862^{*}$ & 1.6 \\
\hline Nicolás Romero & & & & & $112645^{*}$ & 2.3 \\
\hline Tecamac & & & & & $84129^{*}$ & 17 \\
\hline Texcoco & & & & & $105851^{*}$ & 2.1 \\
\hline Chiautla & & & & & $10618^{*}$ & 0.2 \\
\hline Cuauhtitlán- & & & & & & \\
\hline lzcalli & & & & & $173754^{*}$ & 3.5 \\
\hline Cuautitlán- & & & & & & \\
\hline R. Rubio & & & $41156^{*}$ & 1.1 & $-1629 * *$ & 0.0 \\
\hline Teгceт & & & & & & \\
\hline contorno & & & 41156 & 1.1 & 733964 & 14.7 \\
\hline Total & 2173238 & 100.0 & 3756375 & 100.0 & 4991030 & 100.0 \\
\hline
\end{tabular}

Las unidades político-administrativas que más han aportado al crecimiento de la metrópoli de 1950 a 1960 han sido: Gustavo A. Madero, en el primer contorno, que absorbió más del $17 \%$ del crecimiento total y en segundo término Venustiano Carranza y Miguel Hidalgo en el centro. En ese decenio el área central aún colaboraba de manera destacada al crecimiento total de la ZMCM; en cambio, en el siguiente decenio (1960-1970) la situación se modifica y baja bruscamente su participación en el crecimiento total. Gustavo A. Madero siguió manteniendo el mismo peso dentro del total y a esa unidad se agregó Nezahualcóyotl, también perteneciente al primer contorno: entre ambas concentraban casi el $35 \%$ del crecimiento total. Las unidades que le seguían en importancia, aunque con un nivel de participación mucho menor eran Naucalpan, Iztacalco e Iztapalapa, en el mismo contorno, y Tlanepantla en el segundo. Se puede apreciar que en esos diez años, como habíamos anticipado, algunos municipios conurbados comenzaron a colaborar de manera notable al aumento poblacional general de la ZMCM. Por último, en el periodo 1970-1980 dos unidades del 


\section{MAPA III}

Z.M.C.M. CONTORNOS - 1970

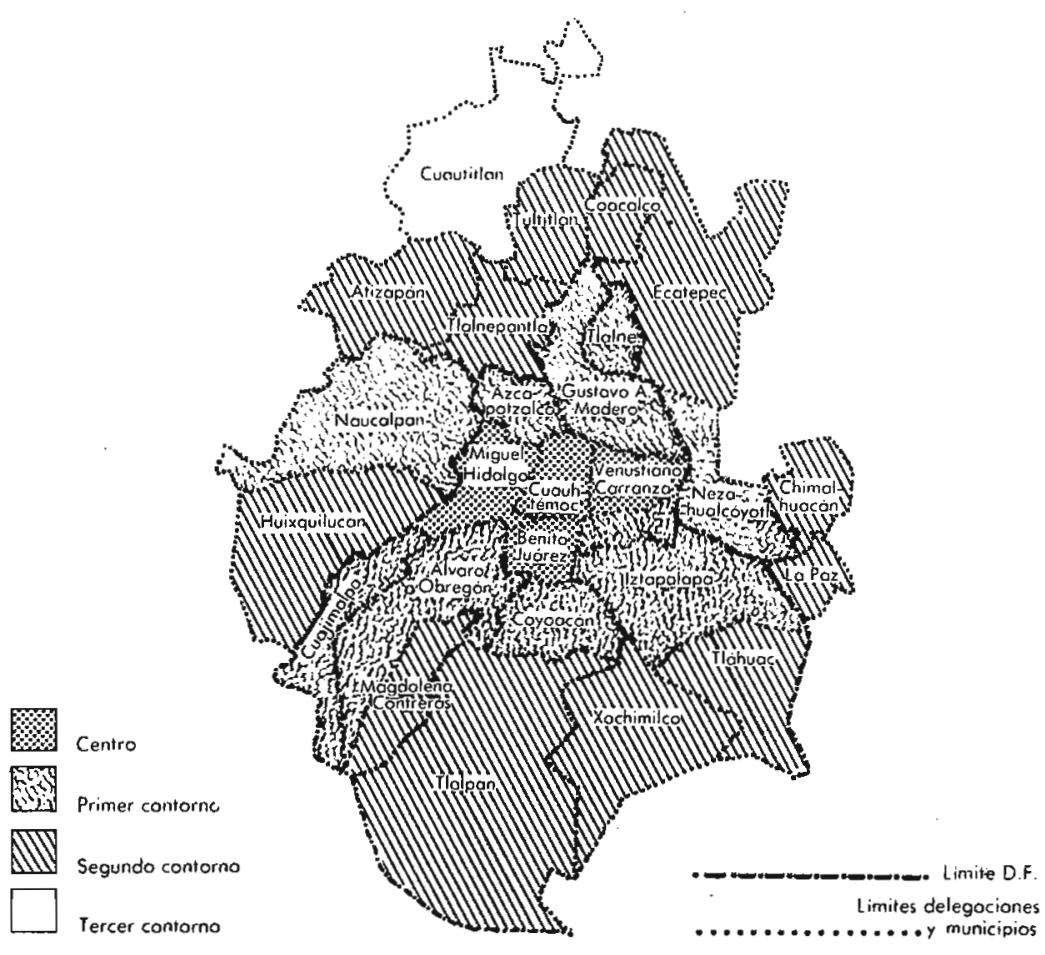


CUADRO 3

Relación análisis centro-periferia con análisis por zonas de consolidación (ZMCM) (1950-1980)

\begin{tabular}{|c|c|c|c|c|c|c|c|c|c|c|c|c|}
\hline \multirow[b]{4}{*}{ Contornos unidades } & \multicolumn{12}{|c|}{ Zonas } \\
\hline & \multicolumn{3}{|c|}{1950} & \multicolumn{3}{|c|}{1960} & \multicolumn{3}{|c|}{1970} & \multicolumn{3}{|c|}{1980} \\
\hline & \multirow[b]{2}{*}{ Zona $^{\star}$} & \multicolumn{2}{|c|}{ Unidades } & \multicolumn{3}{|c|}{ Unidades } & \multirow[b]{2}{*}{ Zona * } & \multicolumn{2}{|c|}{ Unidades } & \multirow[b]{2}{*}{ Zona $^{*}$} & \multicolumn{2}{|c|}{ Unidades } \\
\hline & & Núm. ${ }^{* *}$ & $\%$ & Zona* & Núm. ** & $\%$ & & Núm. ** & $\%$ & & Núm. ** & $\%$ \\
\hline Benito Juárez & 2,3 & & & 1,2 & & & 1 & & 75.0 & 1 & $3 / 4$ & 75.0 \\
\hline Miguel Hidalgo & $1,2,3$ & & & 1,2 & & & 1 & & & 1 & & \\
\hline Cuauhtémoc & 2,3 & & & 3 & & & 1 & & & 1 & & \\
\hline Venustiano Carranza & 4 & & & 3 & & & 2 & & 25.0 & 2 & $4 / 4$ & 25.0 \\
\hline Centro & & & & & & & & & 100.0 & & $4 / 4$ & 100.0 \\
\hline Azcapotzalco & 4 & $3 / 6$ & 50.0 & 3 & $2 / 8$ & 25.0 & 2 & $2 / 9$ & 22.0 & 3 & $5 / 9$ & 55.0 \\
\hline Coyoacán & 5 & $1 / 6$ & 16.7 & 3 & & & 2 & & & 2 & $1 / 9$ & 11.5 \\
\hline Cuajimalpa & & & & 6 & $1 / 8$ & 12.5 & 4 & $1 / 9$ & 11.5 & 4 & $3 / 9$ & 33.5 \\
\hline Gustavo A. Madero & 4 & & & 4 & $4 / 8$ & 50.0 & 3 & $5 / 9$ & $\mathbf{5 5 . 0}$ & 3 & & \\
\hline Iztacalco & 6 & $2 / 6$ & 33.3 & 4 & & & 3 & & & 3 & & \\
\hline Iztapalapa & 6 & & & 4 & & & 3 & & & 4 & & \\
\hline Álvaro Ỏbregón & 4 & & & 4 & & & 3 & & & 3 & & \\
\hline Nezahualcóyotl & & & & & & & 5 & $1 / 9$ & 11.5 & 4 & & \\
\hline Naucalpan & & & & 5 & $1 / 8$ & 12.5 & 3 & & & 3 & & \\
\hline Primer contorno & & $6 / 6$ & 100.0 & & $8 / 8$ & 100.0 & & $9 / 9$ & 100.0 & & $9 / 9$ & 100.0 \\
\hline Magdalena Contreras & 6 & $2 / 2$ & 100.0 & 5 & $1 / 6$ & 17.0 & 5 & $4 / 12$ & 33.3 & 3 & $5 / 13$ & 30.4 \\
\hline Tláhuac & & & & & & & 5 & & & 5 & $3 / 13$ & 23.1 \\
\hline Tlalpan & & & & 4 & $2 / 6$ & 33.0 & 3 & $2 / 12$ & 16.7 & 3 & & \\
\hline Xochimilco & & & & 6 & $3 / 6$ & 53.0 & 4 & $4 / 12$ & 33.3 & 3 & & \\
\hline Atenco & & & & & & & & & & 5 & & \\
\hline Coacalco & & & & & & & 4 & & & 2 & $1 / 13$ & 7.7 \\
\hline Chimalhuacán & & & & 6 & & & 6 & $2 / 12$ & 16.7 & 6 & $1 / 13$ & 7.7 \\
\hline
\end{tabular}




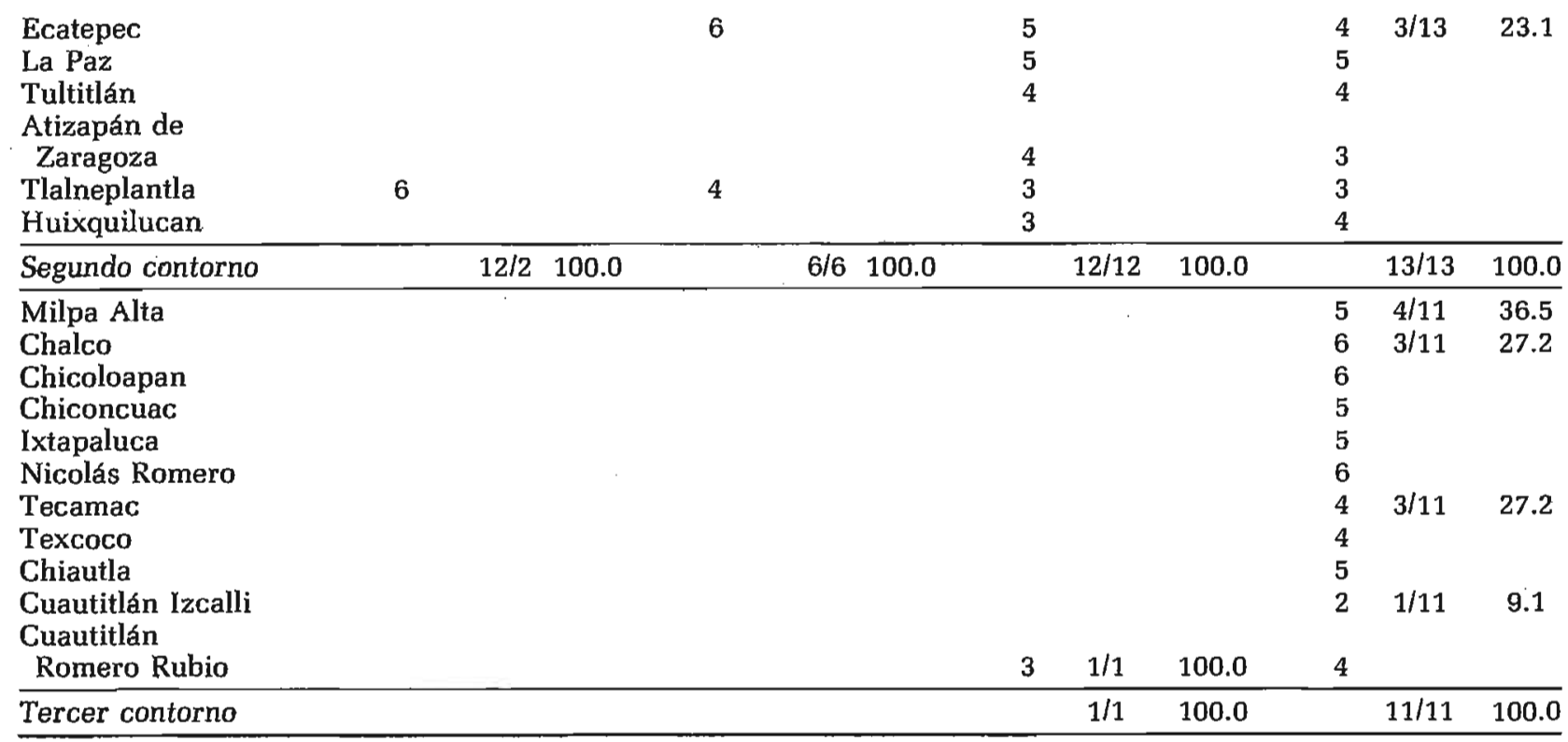

Niveles: 1: alto; 2: medio alto; 3 : medio; 4: medio bajo; 5 : bajo; 6 : muv bajo

* Las zonas de consolidación se tomaron de Rubalcava y Schteingart (1985 y 1987)

** En esta columna se presenta el número de unidades que corresponde a cada nivel de consolidación (en la columna anterior) relacionado con el número total de unidades de cada contorno.

Fuente: Rubalcava y Schteingart, op. cit. 


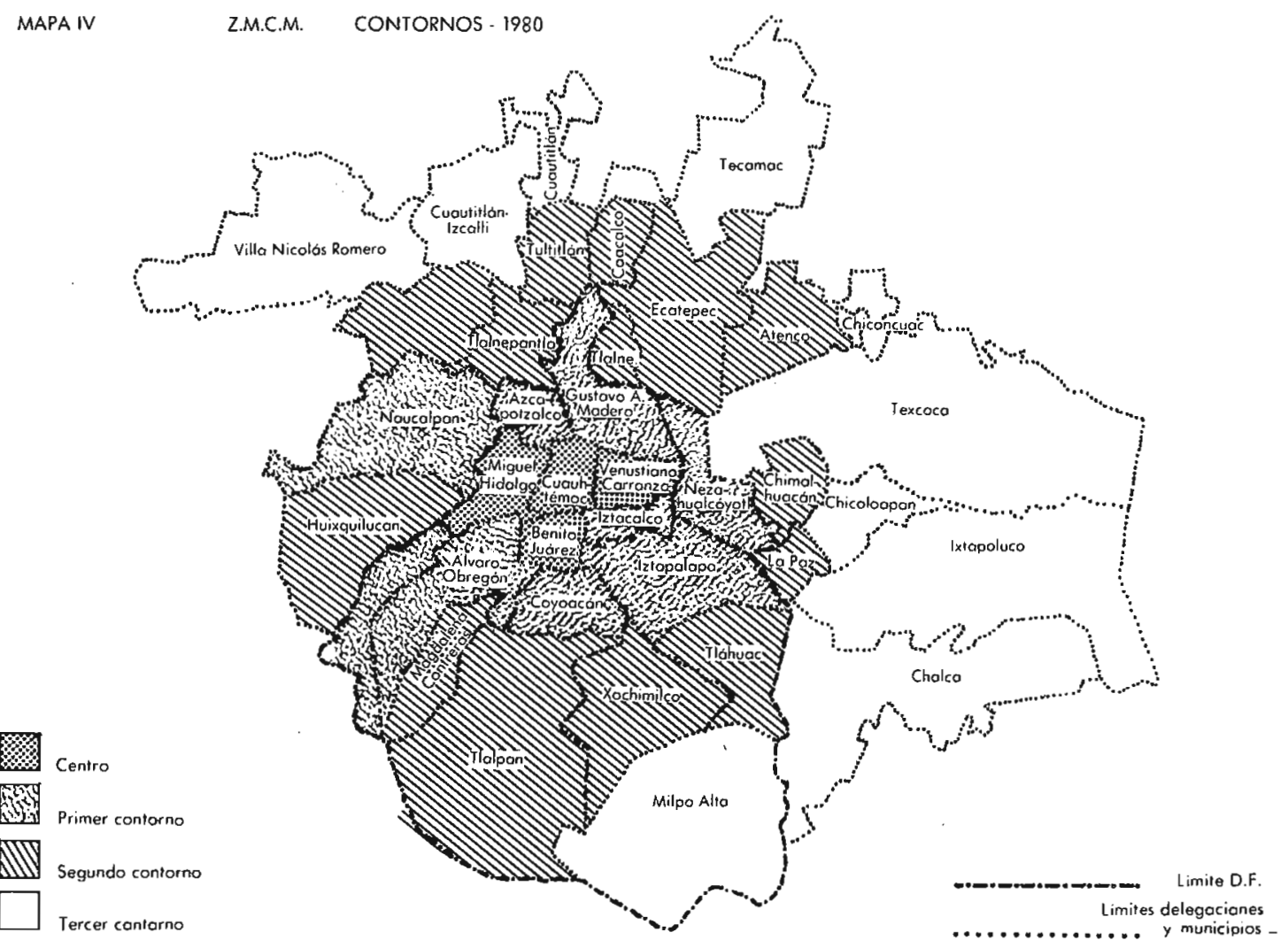


primer contorno aún concentran una parte considerable del incremento total, manteniéndose Nezahualcóyotl [aunque con un peso un poco menor) y cambiando Gustavo A. Madero que es remplazado por Iztapalapa: entre las dos acumulan más del $28 \%$ del total. Casi al mismo nivel de las anteriores, se agrega Ecatepec, en el segundo contorno, y con proporciones bastante menores le siguen Naucalpan y Tlanepantla. Es decir que en esta etapa, con la excepción de Iztapalapa que está en el Distrito Federal, todas las unidades que más aportan al crecimiento urbano se encuentran en el Estado de México, lo que aumenta considerablemente el peso del segundo y tercer contornos.

\section{Crecimiento urbano y niveles de consolidación}

Las unidades que más contribuyeron al crecimiento de la ZMCM en las dos primeras décadas presentaron niveles más altos de consolidación que aquéllás que más contribuyeron a dicho crecimiento durante 1980 (ver cuadro 3). Así, Miguel Hidalgo, Venustiano Carranza y Gustavo A. Madero aparecían con niveles altos y medios ( 2 a 4, según la escala utilizada) en 1950 y 1960; esa última unidad, Iztacalco, Iztapalapa y Tlanepantla, también aparecían con niveles medios ( 3 y 4) en 1960 y 1970 . Vale la pena destacar el caso de Naucalpan, que ascendió rápidamente en esa década al nivel medio (subió dos niveles en la escala, lo cual resulta excepcional, ya que la mayoría de las unidades han ascendido un solo nivel por decenio); en cambio, Nezahualcóyotl se incorporó a un nivel bajo. En 1970-1980, de las tres unidades que concentraban el mayor crecimiento, dos mejoraron, al pasar de un nivel bajo a medio-bajo [Nezahualcóyotl y Ecatepec) mientras la tercera, Iztapalapa, pasó de 3 a 4, bajando de nivel.

Se podría concluir, entonces, que las unidades que han concentrado el mayor crecimiento poblacional, han tendido a presentar en la última década considerada niveles menores de consolidación que en las anteriores, o incluso a bajar de nivel, como ha sucedido con Iztapalapa. Esto se confirma, en cierta medida, si observamos el cuadro 4 donde se presentan los datos relativos a la provisión de agua entubada. Muestran, por ejemplo, que Nezahualcóyotl y Ecatepec se incorporan con niveles bastante inferiores a los de Gustavo A. Madero, en sus momentos de rápida expansión, y que Iztapalapa mejora su provisión de agua de 1970 a 1980, pero en una proporción mennr que las otras unidades del mismo contorno.

¿Por qué ha ocurrido ese fenómeno, que de alguna forma indi- 
ca una degradación de las condiciones en que se da la expansión metropolitana? Si bien este sería un tema para investigar, realizando estudios más puntuales de tipo histórico comparativo, nuestra hipótesis es que algunas nuevas zonas que se abren a la urbanización violenta, para sectores pobres de la población, difícilmente son provistas rápidamente de los medios de consumo colectivo indispensables, a pesar de las luchas sociales que han generado y de algunos programas oficiales de regularización y equipamiento de las mismas. En cambio, en este nivel de análisis se muestra que las unidades político-administrativas que en los años cincuenta y sesenta concentraban parte importante del crecimiento habían comenzado a integrarse a la metrópoli desde mucho tiempo atrás y ya tenían al incorporarse (absorbiendo parte significativa de la expansión urbana) un grado de consolidación que difícilmente una unidad nueva puede adquirir en poco tiempo.

Crecimiento poblacional, consolidación urbana y producción del espacio habitacional

Para explicar cómo la producción de espacios habitacionales que se dan dentro del marco de la legalidad vigente ha incidido en los procesos de consolidación urbana a que hicimos referencia, sobre todo en las unidades que han tenido un papel destacado en la expansión de la metrópoli, introduciremos la información relativa a la aprobación de fraccionamientos; a ella agregaremos algunos datos provenientes de una encuesta aplicada a los promotores inmobiliarios (Schteingart, 1988), en particular aquellos que tienen que ver con la localización de.las operaciones, y que cobrarán una nueva dimensión al ser considerados dentro de un análisis estructural del espacio metropolitano.

De la observación del cuadro 5 podemos concluir que los municipios conurbados del Estado de México que más han colaborado al crecimiento de la ZMCM, han tenido una relación dispar con el desarrollo de los fraccionamientos, lo que indica, en general, la presencia de una urbanización que cumple con los requisitos fijados por la ley, particularmente en lo que se refiere a los servicios básicos para las viviendas. En términos generales, los fraccionamientos incluyen una infraestructura mínimamente aceptable de servicios urbanos, así como viviendas predominantemente para estratos medios de la población, producidas con financiamiento de interés social o bien para sectores de más altos ingresos.

Mientras el crecimiento poblacional de Ecatepec se ha acompañado de la presencia de numerosas viviendas en fracciona: 
CUADRO 4

Porcentaje de viviendas con agua entubada por contorno en la ZMCM [1950-1980)

\begin{tabular}{|c|c|c|c|c|}
\hline Unidad politica & 1950 & 1960 & 1970. & 1980 \\
\hline $\begin{array}{l}\text { Benito Juárez } \\
\text { Cuauhtémoc } \\
\text { Miguel Hidalgo } \\
\text { Venustiano Carranza } \\
\end{array}$ & & & $\begin{array}{l}86.8 \\
82.5 \\
72.2 \\
66.9 \\
\end{array}$ & $\begin{array}{l}91.7 \\
84.8 \\
79.0 \\
72.5 \\
\end{array}$ \\
\hline Centro & 58.9 & 63.2 & 77.1 & 82.0 \\
\hline $\begin{array}{l}\text { Azcapotzalco } \\
\text { Coyoacán } \\
\text { Cuajimalpa } \\
\text { Gustavo A. Madero } \\
\text { Iztacalco } \\
\text { Iztapalapa } \\
\text { Álvaro Obregón } \\
\text { Nezahualcóyotl } \\
\text { Naucalpan } \\
\end{array}$ & $\begin{array}{l}43.1 \\
37.5 \\
\\
40.6 \\
16.2 \\
16.8 \\
34.1\end{array}$ & $\begin{array}{l}52.3 \\
55.2 \\
17.8 \\
50.5 \\
21.4 \\
28.4 \\
34.7 \\
\\
16.1\end{array}$ & $\begin{array}{l}58.3 \\
58.0 \\
33.2 \\
59.5 \\
47.8 \\
47.8 \\
53.4 \\
32.3 \\
52.7 \\
\end{array}$ & $\begin{array}{l}69.7 \\
70.4 \\
48.2 \\
68.9 \\
67.6 \\
56.5 \\
6.1 .6 \\
58.8 \\
63.3 \\
\end{array}$ \\
\hline Primer contorno & 31.4 & 34.5 & 49.2 & 62.8 \\
\hline \multicolumn{5}{|l|}{ Magdalena } \\
\hline $\begin{array}{l}\text { Tláhuac } \\
\text { Tlalpan } \\
\text { Xochimilco } \\
\text { Atenco }\end{array}$ & & $\begin{array}{l}24.3 \\
17.5\end{array}$ & $\begin{array}{l}34.7 \\
54.1 \\
30.8\end{array}$ & $\begin{array}{l}48.4 \\
53.6 \\
59.3 \\
66.4\end{array}$ \\
\hline Coacalco & & & 58.9 & B8.0 \\
\hline Chimalhuacán & & 14.3 & 21.4 & 40.4 \\
\hline Ecatepec & & 6.3 & 38.9 & 63.7 \\
\hline La Paz & & & 24.7 & 50.6 \\
\hline Tultitláṇ & & & 53.4 & 68.4 \\
\hline \multicolumn{5}{|l|}{ Atizapan de } \\
\hline $\begin{array}{l}\text { Zaragoza } \\
\text { Tlalnepantla }\end{array}$ & & & 52.4 & $\begin{array}{l}65.4 \\
67.4\end{array}$ \\
\hline $\begin{array}{l}\text { Tlalnepantla } \\
\text { Huixquilucan }\end{array}$ & 20.6 & 32.2 & $\begin{array}{l}51.4 \\
22.6\end{array}$ & $\begin{array}{l}67.4 \\
50.9\end{array}$ \\
\hline Segundo contorno & 14.5 & 17.3 & 39.4 & 59.6 \\
\hline Milpa Alta & & & & 47.1 \\
\hline Chalco & & & & 57.2 \\
\hline Chicoloapan & & & & 24.9 \\
\hline Chiconcuac & & & & 79.4 \\
\hline Ixtapaluca & & & & 53.5 \\
\hline Nicolás Romero & & & & 34.8 \\
\hline Tecamac : & & & & 50.4 \\
\hline Texcoco & & & & 64.1 \\
\hline Chjautla & & & & 71.5 \\
\hline Cuautitlán-Izcalli & & & & 73.1 \\
\hline Cuautitlán R. Rubio & & & 44.0 & 60.5 \\
\hline Tercer contorno & & & 44.0 & 56.0 \\
\hline
\end{tabular}

Fuente: Censos de Población y Vivienda de 1950, 1960, 1970 y 1980. 
mientos aprobados, ${ }^{2}$ que sobre todo en 1960-1970 absorben una parte muy grande del total aprobado [37.5\%], Nezahualcóyotl presenta un número mucho menor de esas viviendas. A pesar de que ambos municipios exhiben los mismos niveles relativos de consolidación, el cuadro 3 muestra mayores porcentajes de vivienda con agua entubada en el caso de Ecatepec, indicador que seguramente se vincula a un mayor desarrollo de los fraccionamientos. Sin embargo, para entender mejor las diferencias entre las dos unidades político-administrativas, sería necesario considerar con más detalle la historia de su poblamiento, que en el caso de Nezahualcóyotl pasa por un largo periodo de ilegalidad, procesos de regularización, introducción más tardía de servicios, etc. Algunos de esos procesos se han presentado en trabajos anteriores [Schteingart,1981). En cuanto a los otros municipios que ocupan un lugar destacado dentro del crecimiento metropolitano, Naucalpan y Tlanepantla, también tienen un desarrollo importante de fraccionamientos; ambas unidades alcanzan un nivel medio de consolidación, y entre sus fraccionamientos (sobre todo en el caso de Naucalpan) se incluye también un número no despreciable de operaciones residenciales para sectores de mayores ingresos de la población. Aun cuando no se trate de municipios que hayan colaborado de manera destacada al crecimiento de la zona metropolitana, nos referiremos a los casos de Atizapán, Coacalco y Cuautitlán Izcalli, que se han incorporado a niveles altos de consolidación o los han mejorado mucho en el último decenio. Como podemos observar en el cuadro 5 los tres municipios presentan proporciones importantes de viviendas en fraccionamientos aprobados: Coacalco en 1960-1970 y Atizapán y Cuautitlán-Izcalli en 1970-1980 (destacándose sobre todo este último municipio). Coacalco se ha desarrollado básicamente gracias a los grandes fraccionamientos, y dada la escasez de su población, es posible conjeturar que se he urbanizado predominantemente a partir de esta forma de producción de espacios habitacionales. En el caso de Cuautitlán-Izcalli se produce una importante urbanización impulsada por el gobierno del Estado de México; de ahi el gran peso de los fraccionamientos en el conjunto. Los datos de Atizapán, muestran el desa* rrollo de zonas para sectores medio-altos de la población, lo cua] se expresa en el predominio de fraccionamientos residenciales. Justamente la urbanización indicada para esos municipios permi-

\footnotetext{
2 De la información que hemos conseguido sobre fraccionamientos aproba. dos, hemos decidido tomar como mejor indicador de la importancia de los mismos, el número de viviendas previstas, que ellos incluirían. Esto no significa que todas las viviendas ya se hayan construido.
} 
te explicar algunos cambios observados en los grandes lineamientos de la estructuración del espacio en 1970-1980, que se manifiestan en que "el nivel medio-alto de consolidación que en 1970 colindaba con el núcleo más consolidado, se divide en 1980, apareciendo dos de las unidades que lo componen, al norte de la ZMCM, en un área bastante alejada de las anteriores" (Rubalcava y Schteingart, 1987).

Si consideramos ahora la relación, por contorno, entre crecimiento poblacional y desarrollo de los fraccionamientos, podemos concluir que en el primer contorno el crecimiento poblacional ha sido mucho mayor que el de los fraccionamientos, mientras que en el segundo la relación ha sido inversa, con mucho mayor desarrollo de los fraccionamientos en la primera década (por la presencia de Ecatepec y Coacalco). En el tercer contorno se da también la misma relạción a favor de las viviendas en fraccionamientos, pero con mayor desarrollo de fraccionamientos en la segunda década (por la presencia de Cuautitlán-Izcalli).

\section{Algunas consideraciones sobre la producción del espacio} habitacional para sectores medios

La influencia de los fraccionamientos en la expansión de la man cha urbana ha sido considerable, y la forma que ha tomado tuvo que ver con la lógica de operación de los promotores privados y su articulación con las políticas habitacionales del Estado. Entonces el crecimiento poblacional ha sido un factor relevante de la dinámica de esas operaciones inmobiliarias, pero la forma en que se han llevado a cabo ha estado condicionada también por la conformación del sector promocional, y particularmente por el manejo de la tierra, la localización de las promociones y el tipo de financiamiento utilizado, que han constituido importantes elementos mediadores en el asentamiento de la población en distintas partes de la metrópoli.

Es necesario destacar que se dio una gran concentración de fraccionamientos en manos de pocas empresas promotoras, sobre todo cuando comenzó su gran desarrollo en los años sesenta. Algunos grupos, no más de ocho, lograron hacerse en esos años de grandes reservas territoriales y obtener cuantiosas ganancias a partir de la especulación con el suelo. Sin embargo, esa situación empezó a cambiar en los años setenta, cuando la intervención del Estado se incrementó. Los fraccionamientos se hicieron más pequeños, cambiando notablemente la lógica de las operaciones. Así, mientras en 1960-1970 los más grandes fraccionamientos fue- 
CUADRO 5

Crecimiento poblacional de municipios conurbados del estado de México y su relación con las viviendas previstas en fraccionamientos aprobados (1960-1980)

\begin{tabular}{|c|c|c|c|c|c|c|c|c|c|c|}
\hline \multirow[b]{2}{*}{ Municipio } & \multicolumn{5}{|c|}{$1960-1970$} & \multicolumn{5}{|c|}{$1970-1980$} \\
\hline & $\begin{array}{l}\text { Núm } \\
\text { de vi- } \\
\text { viendas }\end{array}$ & $\%$ & $\begin{array}{c}\text { Tipo } \\
\text { de. } \\
\text { fracc. }\end{array}$ & $\begin{array}{l}\text { Crecimien- } \\
\text { to pobla- } \\
\text { cional }^{\star}\end{array}$ & $\begin{array}{c}\% \\
\text { respecto } \\
\text { al total }\end{array}$ & $\begin{array}{c}\text { Núm. } \\
\text { de vi- } \\
\text { viendas }\end{array}$ & $\%$ & $\begin{array}{c}\text { Tipo } \\
\text { de } \\
\text { fracc. }{ }^{\star \star}\end{array}$ & $\begin{array}{l}\text { Crecimien- } \\
\text { to pobla- } \\
\text { cional }^{\star}\end{array}$ & $\begin{array}{c}\% \\
\text { respecto } \\
\text { al total }\end{array}$ \\
\hline Nezahualcóyotl & 25419 & 14.2 & Pop. & 651000 & 38.7 & 18984 & 8.3 & Pop. & 690230 & 24.6 \\
\hline Naucalpan & 15902 & 8.9 & Res. Pop. & 321997 & 19.2 & 20220 & 8.9 & Res. Pop. & 322345 & 11.5 \\
\hline \multicolumn{11}{|l|}{ Primer } \\
\hline Coacalco & 15794 & 8.8 & Pop. & 13197 & 0.8 & 9046 & 4.0 & Pop. & 84156 & 3.0 \\
\hline Chimalhuacán & 857 & 0.5 & Pop. & -57929 & -3.4 & o & 0.0 & & 43005 & 1.5 \\
\hline Ecatepec & 67143 & 37.5 & Pop. & 191811 & 11.4 & 42274 & 18.6 & Pop. & 551821 & 19.6 \\
\hline La Paz & 1834 & 1.0 & Pop. & 34297 & 2.0 & 0 & 0.0 & & 65139 & 2.3 \\
\hline Tultitlán & 8719 & 4.9 & Res. Pop. & 55161 & 3.3 & 11177 & 4.9 & Pop. & 81668 & 2.9 \\
\hline \multicolumn{11}{|l|}{ Atizapán de } \\
\hline Zaragozà & 5652 & 3.2 & Res. Pop. & 47729 & 2.8 & 24044 & 10.6 & Res. Pop. & 154519 & 5.5 \\
\hline Tlalnepantla & 20526 & 11.5 & Res. Pop. & 281930 & 16.8 & 43073 & 18.9 & Res. Pop. & 390796 & 13.9 \\
\hline Huixquilucan & 1627 & 0.9 & Res. R. Camp. & 34604 & 2.1 & 3886 & 1.7 & Res. Pop. & 43545 & 1.6 \\
\hline
\end{tabular}




\begin{tabular}{|c|c|c|c|c|c|c|c|c|c|c|}
\hline $\begin{array}{l}\text { Segundo } \\
\text { contorno }\end{array}$ & 122152 & 68.3 & & 600800 & 35.7 & 133500 & 58.6 & & 1414649 & 50.4 \\
\hline Ixtapaluce & 0 & 0.0 & & 16250 & 1.0 & 2176 & 1.0 & R. Camp. & 41140 & 1.5 \\
\hline Nicolás Romero & 2149 & 1.2 & Pop. & 17887 & 1.1 & & 0.0 & & 65141 & 2.3 \\
\hline Tecamac & 7070 & 4.0 & Res. R. Camp. & 8911 & 0.5 & & 0.0 & & 63247 & 2.3 \\
\hline Cuautitlán & & & & & & & & & & \\
\hline Romero Rubio & 558 & 0.3 & Pop: & 41156 & 2.4 & 1444 & 0.6 & Pop. & -1629 & -0.1 \\
\hline Cuatitlán & & & & & & & & & & \\
\hline Izcalli & 2806 & 1.6 & Pop. & 0 & 0.0 & 51200 & 22.5 & Pop. Res. & 173754 & 6.2 \\
\hline Texcoco & 2779 & 1.6 & Pop. R. Camp. & 23103 & 1.4 & 206 & 0.1 & Pop. & 40223 & 1.4 \\
\hline $\begin{array}{l}\text { Tercer } \\
\text { contorno }\end{array}$ & 15362 & 8.6 & & 107307 & 6.4 & 55026 & 24.2 & & 381876 & 13.6 \\
\hline Total & 178835 & 100.0 & & 1681104 & 100.0 & 227730 & 100.0 & & 2809100 & 100.0 \\
\hline
\end{tabular}

* Basado en el cuadro 2. Se rehicieron los porcentajes tomando como población total sólo la suma correspondiente a los municipios conurbados. ** Los fraccionamientos pueden ser de típo popular, residencial y residencial campestre. Cuando un tipo aparece en cursivas, ello indica su predominio con respecto al otro.

Nota: Se incluyen solamente los municipios donde aparecian fraccionamientos aprobados.

Fuente: Relación de fraccionamientos autorizados por el gobiemo del Estado de México, Dirección de Comunicaciones y Obras Públicas; Departamento de Control y Supervisión de Fraccionamientos. 
ron promovidos por sociedades como FRISA, INFRAPOSA, los grupos Kanner-Ekstein y Bruno Pagliai (que hemos analizado por medio de encuestas aplicadas a los grupos promotores) en los setenta, en cambio, aparecieron como promotoras destacadas ciertas instituciones públicas como el Banco Nacional de Obras y Servicios Públicos (Banobras), el Instituto Nacional de Desarrollo de la Comunidad (Indeco), el Instituto de Acción Urbana e Integración Social (Auris), el Organismo Descentralizado del Estado de México (Odem) y la banca mixta Somex.

Hasta 1980, fecha límite que estamos considerando en este trabajo (ya que nos basamos, para el análisis de la población y la consolidación urbana, en los datos censales), los fraccionamientos autorizados en el Estado de México habian sido promovidos en un $72 \%$ por grupos privados y sólo en un $28 \%$ por organismos públicos; no obstante, algunas de las operaciones más importantes correspondieron a estos últimos, sobre todo al Banobras y al Odem, este último creado para actuar en Cuautitlán-Izcalli. Se ha producido además una articulación entre las acciones públicas y las prácticas privadas que es necesario explicar a fin de comprender la lógica de las operaciones inmobiliarias, evitando la dicotomización entre público y privado que suelen hacer tanto los investigadores como los planificadores urbanos. Esas articulaciones tienen que ver con las relaciones entre las diferentes operaciones o dentro de las mismas. Por ejemplo, en ocasiones son las instituciones públicas las que abren nuevas zonas periféricas a la urbanización (como Villa Coapa y Valle de Aragón, por Banobras); los promotores privados aprovechan la infraestructura y las mejoras introducidas en la zona para establecer sus operaciones inmobiliarias, siguiendo frecuentemente el mismo patrón de urbanización. En otros casos, son las instituciones públicas las que compran terrenos en fraccionamientos promovidos por grupos privados ayudándoles, de esta manera, a financiar parte de sus operaciones o a recuperar lo invertido, sobre todo en momentos de contracción del mercado.

Podemos concluir que, en general, la intervención del Estado en el desarrollo de los espacios habitacionales, no necesariamente ha implicado una planeación racional del espacio metropolitano, ya que con frecuencia ha seguido la lógica impuesta por los promotores privados. La forma en que se han elegido los terrenos y localizado las operaciones ha dependido de las características del mercado del suelo, que en algunos municipios ha estado totalmente dominado por algunos grupos inmobiliarios.

El impacto de los fraccionamientos en la expansión metropolitana varía según la unidad político-administrativa considerada, 
y resulta poco significativo en aquéllas que más han concentrado el crecimiento total de la ZMCM. En el caso de Iztapalapa ello resulta muy claro, por la baja relativa de su nivel de consolidación, e incluso en Ecatepec, donde, a pesar de la gran cantidad de fraccionamientos consignada, el área ocupada por asentamientos irregulares parece tener un peso nada despreciable (alrededor del $20 \%$ de la superficie de uso habitacional del municipio; Iracheta, 1984).

Por otra parte, la población beneficiada por los programas de vivienda terminada, realizados con el apoyo financiero de los organismos más importantes que actúan en la ZMCM, entre 1963 y 1980 , representa aproximadamente el $15.5 \%$ del crecimiento de la población de esa zona durante el periodo (ver cuadros 1 y 6). Esto confirma las grandes limitaciones de las acciones estatales dentro del sector llamado "formal" de vivienda, y permite comprender, en parte, la gran expansión de los asentamientos irregulares que veremos a continuación.

\section{Los asentamientos irregulares y la conformación del espacio}

No existen fuentes censales o estadísticas adecuadas para conocer con precisión la proporción de habitantes que ocupan estos asentamientos, y se han presentado a menudo datos bastante contradictorios. Hacia 1952 se estimaba que un $22 \%$ de los habitantes de la ZMCM habitaba colonias populares y hacia 1976 los cálculos fueron de un $50 \%$. Esas colonias cubrían un $64 \%$ de las áreas urbanizadas de la metrópoli (Connolly, 1981).

Algunos cálculos más recientes [Iracheta, 1987) consideran

CUADRO 6

Viviendas producidas por los princiaples organismos públicos en la ZMCM

\begin{tabular}{lrrrr}
\hline & $1963-1980$ & $1963-1970$ & $1971-1976$ & $1977-1980$ \\
\hline BANOPSA & 37483 & $23 \cdot 880$ & 13603 & - \\
FOVI & 69392 & 33953 & 28793 & 6646 \\
DDF/DGHP & 52461 & 13000 & 39461 & - \\
INV/INDECO & 24745 & 3996 & 20749 & - \\
ISSSTE/FOVISSSTE & 36768 & 17790 & 8212 & 10766 \\
INFONAVIT & 51338 & - & 22932 & 28406 \\
\hline Total & 272187 & 92619 & 133750 & 45818 \\
\hline Promedio anual & 15122 & 11577 & 22292 & 11455 \\
\hline
\end{tabular}

Fuente: hasta 1976, G. Garza y M. Schteingart. "La acción habitacional del Estado de Méxíco"; de 1976 a 1980 , datos de cada organismo. 
que en el Distrito Federal existían en 1986 unos $111 \mathrm{~km}^{2}$ de terrenos irregulares, los cuales representan el $25 \%$ del área urbana dedicada a uso habitacional dentro del Distrito Federal, siendo las delegaciones que presentan mayor grado de irregularidad: Iztapalapa, Gustavo A. Madero, Tlalpan, Álvaro Obregón, Tláhuac e Iztacalco (en el primero y segundo anillos]. En esa superficie se han considerado unos 300000 lotes para aproximadamente 1.6 millones de habitantes, lo que representa alrededor del $15 \%$ de la población de la capital del país. Sin embargo, se aclara que esas cifras son muy bajas porque también habría que incluir los terrenos en proceso de regularización y que las fuentes utilizadas consideran separadamente; si se agregan esos datos a los anteriores se llegaría a un total de $212 \mathrm{~km}^{2}$ y a unos 540000 lotes con alrededor de 3 millones de habitanies. En 17 municipios conurbados de] Estado de México, para el mismo año, se consideraban alrededor de 140 $\mathrm{km}^{2}$ de terrenos irregulares [el $35 \%$ del área de uso habitacional de esas unidades] incluyendo unos 444000 lotes para alrededor de 2.6 millones de habitantes, que representan el $34 \%$ del total de población de esos municipios. Probablemente las cifras referidas a la irregularidad sean bastante menores que las consignadas para 1976 porque aquéllas estaban considerando a todos los asentamientos que comenzaron como irregulares y que por tanto tuvieron procesos distintos que los de los fraccionamientos aprobados antes de la urbanización y la construcción de viviendas. Aun cuando se hayan beneficiado posteriormente con la regularización, a nuestro criterio ese cálculo es más pertinente para diferenciar los distintos procesos, legales e ílegales, que están al inicio de la formación de los espacios habitacionales.

Los rasgos comunes de los asentamientos ílegales son, en su origen, la irregularidad en la tenencia de la tierra, la falta de servicios, la precariedad de las viviendas y la imposibilidad de sus residentes de ser sujetos de crédito de la mayor parte de los programas de vivienda del Estado. Pero junto a estos rasgos comunes aparece toda una gama de situaciones distintas, que implican la presencia de diferentes tipos de agentes y relaciones sociales, diferentes grados de irregularidad, condiciones de vida, formas habitacionales y posibilidades de superar las precarias características iniciales del asentamiento. La población involucrada pertenece, en parte, a la clase obrera, sobre todo a los sectores de la misma que reciben salarios más bajos y no tienen asistencia oficial para la obtención de su vivienda; en parte también a los grupos de subempleados y desempleados. Como se muestra en el cuadro 7 , en el que se presentan los ingresos de la población (según datos de los censos) ordenados por contornos, podemos comprobar que en 
general, en 1970, han predominado los ingresos bajos (hasta una vez el salario mínimo) en los tres contornos considerados, mientras en 1980 se presenta una mejoría para el primero y segundo contornos y una situación más negativa para el tercero, por la incorporación de nuevas unidades en las que predominan ampliamente las familias de bajos ingresos. A pesar de que prácticamente todas las unidades político-administrativas han mejorado su situación entre 1970 y 1980, podemos notar que todavía algunas unidades del primer contorno (Cuajimalpa, Iztapalapa y Nezahualcóyotl), casi el $40 \%$ de las unidades del segundo contorno y más del $60 \%$ de las del tercero, presentan a más del $50 \%$ de las familias que reciben ingresos de hasta una vez el salario mínimo. Estas proporciones mayores, sobre todo en Chimalhuacán, La Paz y Chalco, coinciden con municipios donde casi no se han aprobado fraccionamientos y donde el desarrollo de asentamientos irregulares ha sido mayor. Es decir que los bajos ingresos son, en cier. ta medida, un indicador de que amplios sectores de la población no pueden sino tener acceso a los asentamientos irregulares.

Se han presentado varias clasificaciones de los asentamientos irregulares, entre las cuales hemos optado por elegir la que establece dos tipos más frecuentes: los fraccionamientos ilegales y las colonias de invasión. Los primeros fueron promovidos por propietarios individuales, por empresas promotoras o por los comisariados ejidales. La tierra fue dividida en lotes y se vendió casi sin servicios (a veces sólo con electricidad y unas pocas tomas de agua). Son ilegales porque no cumplen con los reglamentos de construcción y urbanización, y también porque la propiedad del suelo es confusa o porque se vende tierra que por ley no puede ser comercializada, como los ejidos. Frecuentemente los fraccionadores no tienen título de propiedad de la tierra que comercializan, y a menudo venden varias veces el mismo lote. Las colonias de invasión han disminuido en los úitimos años, aunque en ocasiones se combinan diferentes modalidades de ocupación en un solo asentamiento. No existen suficientes investigaciones que puedan dar cuenta de la frecuencia de las distintas formas de ocupación de estos terrenos, pero parece existír consenso en cuanto a que la forma más frecuente, sobre todo más recientemente, es la compra (sobre tọdo de terrenos ejidales) y no la invasión.

En el caso de la ZMCM los fraccionamientos ilegales de terrenos públicos se han ubicado, principalmente, en la dirección noreste de la misma, en las tierras producto de la desecación del lago de Texcoco, siendo el ejempîo más típico el de Nezahualcóyotl. Los fraccionamientos de terrenos ejidales se ençuentran particularmente en el poniente, norponiente y sur de la zona metropolita- 
CUADRO 7

Ingreso de la población en la ZMCM [1970-1980]

\begin{tabular}{|c|c|c|c|c|c|c|c|}
\hline \multirow{2}{*}{$\begin{array}{l}\text { Unidades } \\
\text { políticas }\end{array}$} & \multicolumn{3}{|c|}{1970} & \multicolumn{3}{|c|}{1980} & \multirow{2}{*}{$\frac{\text { Tendencia }}{70-80}$} \\
\hline & Bajos & Medios & Altos & Bajos & Medios & Altos & \\
\hline Benito Juárez & 39.7 & 48.4 & 11.9 & 29.9 & 64.0 & 6.0 & \multirow{5}{*}{$\begin{array}{l}\text { Medio } \\
\text { a } \\
\text { medio }\end{array}$} \\
\hline Cuauhtémoc & 40.5 & 53.9 & 5.6 & 35.2 & 62.6 & 2.1 & \\
\hline Miguel Hidalgo & 46.2 & 45.3 & 8.6 & 41.0 & 53.1 & 5.0 & \\
\hline \multicolumn{7}{|l|}{ Venustiano } & \\
\hline & 45.8 & 51.3 & 2.9 & 41.3 & 57.8 & 0.7 & \\
\hline Centro & 43.0 & 49.7 & 7.2 & 36.8 & 59.4 & 3.6 & \\
\hline Azcapotzalco & 45.5 & 17.0 & 3.2 & 37.6 & 60.9 & 1.4 & \multirow{10}{*}{$\begin{array}{l}\text { Bajo } \\
\text { a } \\
\text { medio }\end{array}$} \\
\hline Coyoacán & 48.0 & 42.1 & 9.9 & 38.5 & 56.6 & 4.9 & \\
\hline Cuajimalpa & 62.9 & 35.7 & 1.4 & 56.9 & 40.7 & 1.8 & \\
\hline Gustavo A. & & & & & & & \\
\hline Madero & 48.1 & 48.8 & 3.1 & 42.0 & 56.9 & 1.0 & \\
\hline Iztacalco & 53.4 & 44.1 & 2.8 & 45.2 & 53.7 & 1.6 & \\
\hline Iztapalapa & 54.0 & 43.4 & 2.6 & 50.2 & 48.9 & 0.8 & \\
\hline Álvaro Ỏbregón & 52.7 & 40.3 & 7.0 & 45.3 & 50.2 & 4.5 & \\
\hline Nezahualcóyotl & 62.1 & 36.8 & 1.1 & 56.2 & 43.3 & 0.7 & \\
\hline Naucalpan & 50.5 & 37.4 & 12.1 & 45.0 & 48.5 & 6.5 & \\
\hline \multicolumn{8}{|l|}{ Primer } \\
\hline contorno & 53.0 & 42.2 & 4.8 & 46.3 & 51.1 & 23.2 & \\
\hline \multicolumn{8}{|l|}{ Magdalena } \\
\hline Contreras & 60.6 & 39.7 & 0.3 & 48.4 & 47.6 & 4.1 & \multirow{14}{*}{$\begin{array}{c}\text { Bajo } \\
\text { a } \\
\text { equilibrio }\end{array}$} \\
\hline Tláhuac & 66.1 & 32.9 & 1.0 & 57.8 & 41.9 & 0.2 & \\
\hline Tlalpan & 46.7 & 47.8 & 5.5 & 44.1 & 51.4 & 4.4 & \\
\hline Xochimilco & 63.1 & 35.6 & 1.3 & 49.5 & 48.7 & 1.7 & \\
\hline Atenco & & & & 66.6 & 33.1 & 0.1 & \\
\hline Coacalco & 48.7 & 48.9 & 2.5 & 27.7 & 70.7 & 1.5 & \\
\hline Chimalhuacán & 73.5 & 25.7 & 0.7 & 70.3 & 29.4 & 0.3 & \\
\hline Ecatepec & 54.3 & 44.3 & 1.4 & 46.8 & 52.7 & 0.6 & \\
\hline La Paz & 66.8 & 32.4 & 0.8 & 63.7 & 36.0 & 0.2 & \\
\hline Tultitlán & 44.6 & 53.3 & 2.1 & 42.8 & 56.5 & 0.6 & \\
\hline \multicolumn{7}{|l|}{ Atizapán de } & \\
\hline Zaragoza & 50.3 & 40.5 & 9.2 & 39.8 & 52.9 & 7.2 & \\
\hline Tlanepantla & 47.3 & 47.7 & 5.0 & 41.5 & 56.0 & 2.4 & \\
\hline Huixquilucan & 74.9 & 22.7 & 2.4 & 58.2 & 36.4 & 5.4 & \\
\hline \multicolumn{6}{|l|}{ Segundo } & 2.2 & \\
\hline
\end{tabular}

na y han surgido como corolario de arreglos entre ejidatarios, comuneros y funcionarios de la Secretaría de la Reforma Agraria. En repetidas ocasiones es a partir de la creación de una zona urbana ejidal que comienzan las ventas ilegales y la formación de 
CUADRO 7 (continuación)

\begin{tabular}{|c|c|c|c|c|c|c|c|}
\hline \multirow{2}{*}{$\begin{array}{l}\text { Unidades } \\
\text { políticas }\end{array}$} & \multicolumn{3}{|c|}{1970} & \multicolumn{3}{|c|}{1980} & \multirow{2}{*}{$\frac{\text { Tendencia }}{70-80}$} \\
\hline & Bajos & Medios & Altos & Bajos & Medios & Altos & \\
\hline Milpa Alta & & & & 69.6 & 30.2 & 0.1 & \\
\hline Chalco & & & & 75.7 & 23.9 & 0.3 & \\
\hline Chicoloapan & & & & 71.0 & 28.7 & 0.2 & \\
\hline Chiconcuac & & & & 76.1 & 23.5 & 0.4 & \\
\hline Ixtapaluca & & & & 66.2 & 33.4 & 0.3 & Bajo \\
\hline \multicolumn{8}{|l|}{ Nicolás } \\
\hline Romero & 58.1 & 41.5 & 0.4 & 47.1 & 33.7 & 0.3 & a \\
\hline Tecamac & 52.2 & 46.4 & 1.4 & 44.7 & 39.7 & 1.2 & bajo \\
\hline Texcoco & & & & 60.3 & 38.6 & 1.1 & acentuado \\
\hline Chiautla & & & & 74.4 & 25.2 & 0.2 & \\
\hline \multicolumn{8}{|l|}{ Cuautitlán } \\
\hline Izcalli & & & & 33.4 & 64.7 & 1.7 & \\
\hline Cuautitlán & & . & & & & & \\
\hline Romero Rubio & 56.8 & 41.2 & 2.0 & 48.1 & 51.1 & 1.7 & \\
\hline Tercer contorno & 55.7 & 43.0 & 1.2 & 60.6 & 35.7 & 0.7 & \\
\hline
\end{tabular}

este tipo de colonias. Algunos estudios han mostrado que alrededor del.42\% de los asentamientos populares de la ZMCM se desarrolló en ejidos (Iracheta et al., 1984). Mientras aquéllos han tendido a incrementarse, los fraccionamientos ilegales de terrenos privados o públicos se han vuelto menos frecuentes, debido a las restricciones impuestas por el Estado.

\section{Conclusiones}

Este trabajo ha intentado explicar algunas tendencias del crecimiento y la distribución de la población, y de la estructuración del espacio en la ZMCM, relacionando datos censales, que han servido como indicadores para medir los procesos de crecimiento y consolidación urbana (a nivel de las unidades político-administrativas que forman parte de la ZMCM y de los contornos que se constituyen con la adición de esas unidades) con la información obtenida de organismos oficiales y de nuestros propios trabajos de campo referidos a la producción del espacio habitacional. Las conclusiones 
más importantes que han surgido del análisis son las siguientes.

1. Algunas de las unidades político-administrativas que más peso han tenido en el crecimiento poblacional de la ZMCM se han ido desplazando del centro, en la década 1950-1960, al primer contorno en la década siguiente y al segundo contorno en 19701980. Con esto, ciertos municipios conurbados del Estado de México, como Nezahualcóyotl, Ecatepec, Naucalpan y Tlanepantla se han convertido, a partir de los años sesenta, en unidades de fuerte dinámica poblacional, que han concentrado una parte muy importante del crecimiento metropolitano.

2. Las unidades que han contribuido en mayor medida a ese crecimiento poblacional en las dos primeras décadas han tendido a presentar mayores niveles de consolidación que las unidades que más colaboraron durante la última década, encontrándose además un caso [Iztapalapa, en el Distrito Federa]) que ha bajado de nivel. Ello puede explícarse, por un lado, debido a la aceleración del ritmo de la expansión urbana en algunas partes del territorio de la ZMCM y, por otro, a que las nuevas zonas que se abren bruscamente a la urbanización, para estratos de la población con muy débiles ingresos, difícilmente pueden contar a corto plazo con los servicios y equipamientos que permiten la consolidación urbana. Esto ocurre a pesar del mejoramiento que, según hemos podido comprobar, han experimentado algunos indicadores sociales durante los años setenta, y que seguramente no ha continuado en los ochenta, sobre todo a partir de la crisis económica.

3. Al comparar el peso del crecimiento poblacional de los distintos municipios conurbados del Estado de México, donde se ha concentrado en mayor medida la expansión metropolitana, con la proporción de fraccionamientos aprobados, y en particular con las viviendas previstas en tal forma de urbanización, se observa la existencia de una relación dispar entre esos porcentajes. Nezahualcóyotl exhibe la situación más desventajosa, ya que mientras representa el 39 y $25 \%$ del crecimiento total de la población en 1960-1970 y 1970-1980, respectivamente, sólo ha concentrado el 14 y $8 \%$ de las viviendas en fraccionamientos aprobados en esas mismas décadas. Ecatepec, en cambio, presenta una relación muy distinta, ya que en 1960-1970 ha concentrado el 37\% de los fraccionamientos, con un crecimiento poblacional del 11\%; sin embargo, esa situación cambia notablemente en la década siguiente, ya que su gran crecimiento no parece haberse acompañado de un desarrollo similar de los fraccionamientos. En el otro extremo, algunos municipios, como Coacalco y Cuautitlan Izcalli, que no han tenido un peso importante en el crecimiento metropolitano, han absorbido proporciones relativamente fuertes de viviendas previs- 
tas en fraccionamientos aprobados, situación que se expresa en el alto nivel de consolidación alcanzado.

4. La concentración y el desarrollo de fraccionamientos en algunos municipios han tenido que ver con las prácticas de los promotores inmobiliarios privados, sobre todo con el acaparamiento de terrenos en ciertas zonas por parte de algunas empresas, pero también con la combinación que se ha dado, en distintos momentos, entre las prácticas de esos agentes privados y las políticas de instituciones estatales dedicadas al financiamiento y la promoción de la vivienda. Entre estas últimas destacan el Infonavit, Banobras y Somex.

5. A pesar del número importante de fraccionamientos aprobados y de la considerable superficie que ocupan, incluso en las unidades que más han colaborado al crecimiento metropolitano, los asentamientos irregulares tienen una presencia destacada dentro de los espacios habitacionales de esas unidades. Si bien Nezahualcóyotl es el municipio donde la irregularidad se hizo más. patente (lo cual resulta coherente con lo que señalamos con relación al escaso número de fraccionamientos aprobados] también Naucalpan, Tlanepantla y Ecatepec, en el primero y segundo contornos, con muchos fraccionamientos aprobados, presentan un área importante (que oscila aproximadamente entre el 20 y el $40 \%$ de su superficie dedicada a uso habitacional), ocupada por esos asentamientos irregulares.

6. El presente estudio nos ha permitido mostrar sistemáticamente cómo se han dado algunas tendencias generales del desarrollo poblacional y socioespacial de la ZMCM; sin embargo, las explicaciones que hemos esbozado de algunos fenómenos observados resultan todavía insuficientes para dar cuenta de los complejos procesos sociales que están detrás de las formas espaciales. Se requerirían muchos más estudios, sobre todo a nivel de las unidades político-administrativas más importantes, para poder avanzar en el plano explicativo mencionado.

\section{Bibliografia}

Connolly, Priscilla [1981]. "La autoconstrucción espontánea: ¿̇solución o problema?", en Investigaciones en autoconstrucción, México, Consejo Nacional de Ciencia y Tecnología.

Iracheta, Alfonso (coord.) (1984). El suelo, recurso estratégico para el desarrollo urbano, Universidad Autónoma del Estado de México.

Iracheta, Alfonso (1987). "Algunas reflexiones en relación al problema 
del suelo para la vivienda en la Zona Metropolitana de la Ciudad de México", mimeo.

Negrete, M. Eugenia y H. Salazar (1986). "Zonas metropolitanas en México, 1980", en Estudios Demográficos y Urbanos, vol. 1, núm.1, eneroabril.

Rubalcava, R. María y M. Schteingart (1985). "Diferenciación socioespacial intraurbana en el Área Metropolitana de la Ciudad de México", en Estudios Sociológicos, vol. 3, núm. 9, septiembre-diciembre.

Rubalcava, R. María y M. Schteingart (1987]. "Estructura urbana y diferenciación socio-espacial en la zona metropolitana de la ciudad de México (1970-1980)", en Atlas de la Ciudad de México, Departamento del Distrito Federal y El Colegio de México.

Schteingart, Martha (1981). "Formación y consolidación de un asentamiento popular en México. El caso de Ciudad Nezahualcóyotl", en Revista Interamericana de Planificación, México, núm. 57.

(1988). "Producción habitacional y promoción inmobiliaria en la zona metropolitana de la ciudad de México, en Procesos habitacionales en la ciudad de México, M. Michel, coordinador, Sedue, uam, México. 